\title{
Design Optimization of Building Structures Using a Metamodeling Method
}

\author{
Qian Wang, Lucas Schmotzer, Yongwook Kim \\ Department of Civil and Environmental Engineering, Manhattan College \\ 4513 Manhattan College Parkway, Riverdale, NY 10471, USA \\ qian.wang@institution.org; 1schmotzer01@manhattan.edu; yongwook.kim@manhattan.edu
}

\begin{abstract}
Numerical optimization techniques have been widely used in the design of different structural and mechanical engineering systems. For practical building structures, finite element (FE) analyses are required in order to obtain structural responses, such as stresses, forces, and deformations. In a nested application of design optimization, an FE code shall be integrated with an optimization algorithm; therefore, software input/output and user-interface programming are necessary. In this work, an alternative approach was pursued. A metamodeling method based on augmented radial basis functions (RBFs) was used to approximate structural responses so that the FE software was replaced by an approximate model, i.e., metamodel. It was not required to directly integrate the FE code in the numerical optimization loop. Once the explicit response functions became available, a traditional gradient-based algorithm was applied so that an optimal design could be found. The overall procedure of the design optimization problem was presented. As an illus trative example, a three-dimensional (3D) reinforced concrete (RC) building was studied and the optimization objective was determined to reduce the torsional responses of the building. Detailed FE analyses were first performed to obtain the building responses at the sample points, before the metamodel was constructed and optimization algorithm was applied. The optimization method worked well and provided a useful tool for practicing engineers.
\end{abstract}

Keywords: Structural Optimization, Finite Element (FE), Metamodeling, Radial Basis Functions (Rbfs), Reinforced Concrete (RC) Building, Torsional Responses.

\section{Introduction}

Structural optimization methods and applications have been extensively studied in the last few decades, and detailed reviews can be found in literature [1,2]. More work is still needed for the application of design optimization to large-scale building structures $[3,4]$. Structural design optimization can be achieved by integrating structural analyses with an iterative optimization procedure. The traditional gradient-based optimization algorithm requires structural design sensitivity analysis, due to the implicit nature of structural response functions in terms of design variables [2]. Moreover, sensitivity analysis requires the coupling of $\mathrm{FE}$ analyses and software input/output programming.

Instead of directly employing expensive structural analyses in numerical optimization loops, a metamodel such as a response surface can be adopted in the optimization process. A metamodeling technique uses an approximate function to replace the actual response function [5,6]. In addition to the traditional response surface methods, other metamodeling approaches are also available, including kriging and RBFs [7-9]. One advantage of RBF metamodels is that there are no errors at any sample point. Recent research showed that RBFs could be used to generate very accurate approximation functions. However, metamodeling methods integrated with design optimization techniques have not been widely adopted in civil structural design. There is a need to apply these methods to the design of practical building structures.

In this study, a metamodeling approach based on augmented RBFs was studied and design optimization was performed using a traditional gradient-based algorithm. The metamodeling approach became very efficient since the response function was explicit in terms of design variables. An existing FE analysis code could be easily used. As an illustrative example, a 3D RC building was optimized to reduce the torsional responses of the building. Two different optimization formulations were studied and optimization results were obtained using the proposed approach. For the remaining of the paper, the $\mathrm{RBF}$ technique is introduced to create the explicit functions of structural responses of the building, before the optimization problem is outlined. Finally, the numerical example and some concluding remarks are presented. 


\section{The Optimization Problem}

\subsection{Design Optimization Formulation}

A general design optimization problem is to find a vector of design variables, $\mathrm{x}$, in order to minimize an objective function

$$
C(\mathbf{x})
$$

subject to

$$
\begin{gathered}
\mathrm{g}(\mathbf{x}) \leq \mathbf{0} \\
\mathbf{x}^{L} \leq \mathbf{x} \leq \mathbf{x}^{U}
\end{gathered}
$$

Equation (2) defines design constraint functions $\mathbf{g}(\mathbf{x})$, which include all structural design requirements for a given problem. Equation (3) specifies the upper and lower limits of the design variables.

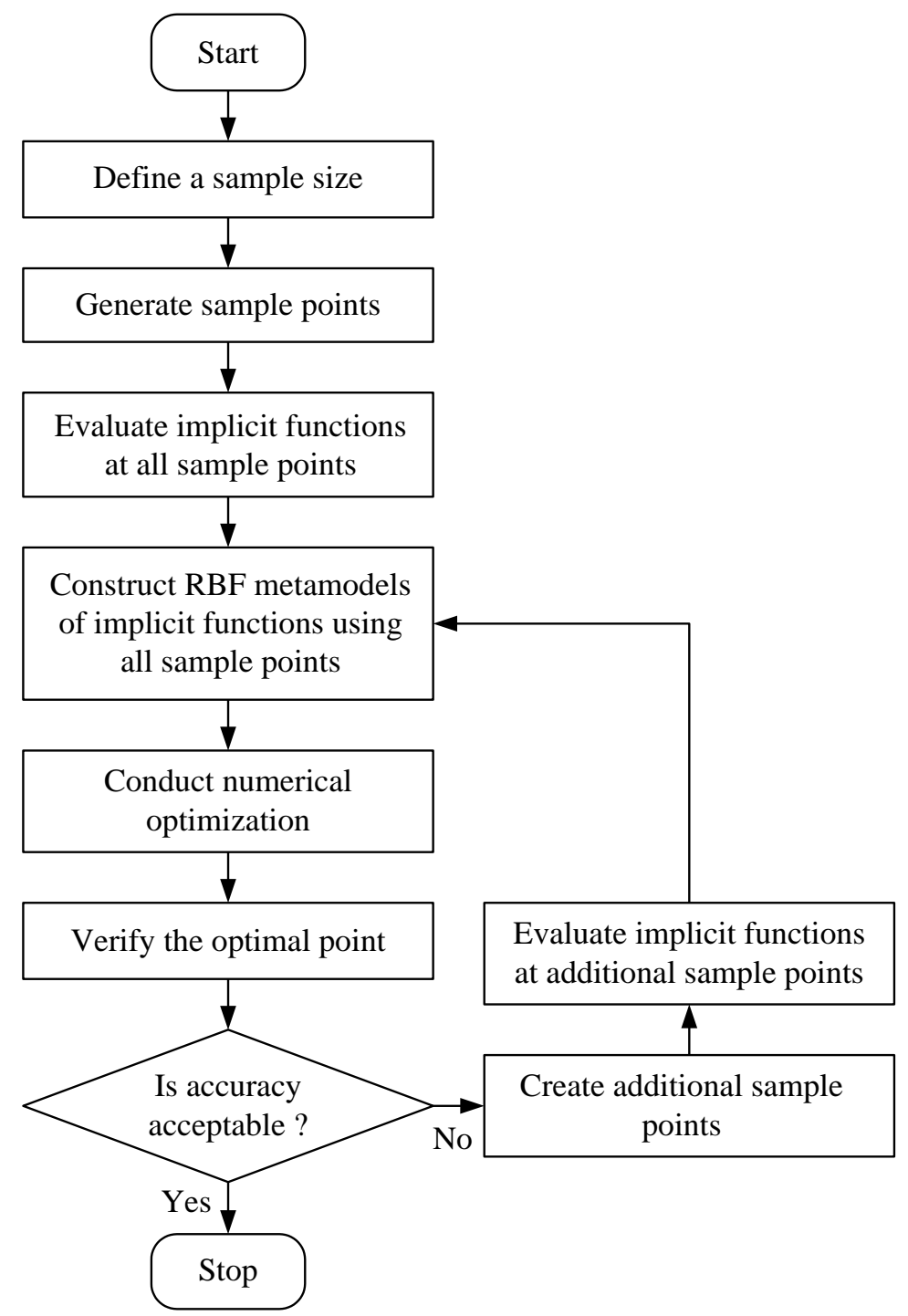

Fig. 1: Flowchart of design optimization using RBF Metamodels. 


\subsection{Metamodels Based on Augmented RBFs}

For a response function $\mathrm{g}(\mathbf{x})$, an RBF metamodel of $\mathrm{g}(\mathbf{x})$ can be written as [8,9]:

$$
\tilde{g}(\mathbf{x})=\sum_{i=1}^{n} \lambda_{i} \phi\left(\left\|\mathbf{x}-\mathbf{x}_{i}\right\|\right)
$$

where $n$ is the number of sample points, $\phi$ is a basis function, and $\lambda_{i}$ is the corresponding coefficient, respectively. An augmented RBF model can be obtained, when $\widetilde{\mathrm{g}}(\mathbf{x})$ in Eq. (4) is augmented with a polynomial function [8,9].

\subsection{Overall Optimization Procedure}

The overall procedure of the design optimization method is highlighted in Fig. 1. Once the initial sample points are generated, FE analyses are conducted. RBF-metamodels are constructed using all the samples. Numerical optimization can be executed using any optimization algorithm. In this research, the solver in Microsoft Excel 2013 [10] was used as the optimization engine. The optimal design can be further verified based on the FE analysis results at the optimal design point. If the accuracy of a metamodel needs to be improved, additional sample points can be generated. Therefore more FE analyses should be conducted.

\section{One Illustrative Example}

To illustrate the optimization method, the design of a hypothetical seventeen-story RC building is considered. A typical floor plan of the RC building is shown in Fig. 2. The building has two towers and they are connected on top of the building. Three design variables $w_{1}, w_{2}$, and $w_{3}$ were adopted in this example, representing three different shear wall thicknesses. The lower and upper limits of all the three variables were $0.4 \mathrm{~m}$ and $0.8 \mathrm{~m}$. To reduce the torsional responses of the building, the design objective was determined as the ratio of the first torsional and translational modal periods.
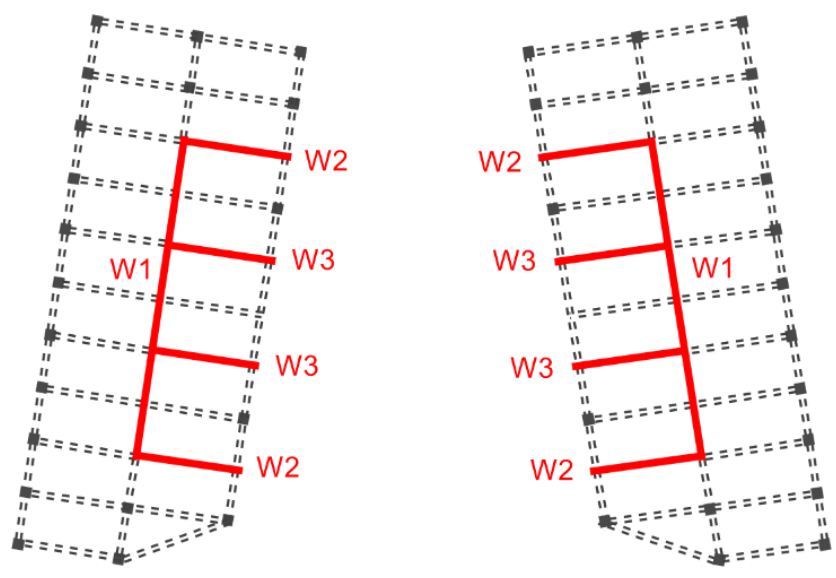

Fig. 2: A typical floor plan of an RC building.

Two optimization formulations were considered. The first optimization formulation is to minimize:

subject to

$$
C\left(w_{1}, w_{2}, w_{3}\right)=\frac{T_{t}}{T_{1}}
$$

$$
0.4 m \leq w_{1}, w_{2}, w_{3} \leq 0.8 m
$$

where $T_{t}$ and $T_{l}$ are the first torsional and translational periods from modal analysis, respectively. The second optimization formulation is to minimize the total cross-sectional area of shear walls: 
subject to

$$
C\left(w_{1}, w_{2}, w_{3}\right)=\sum_{i=1}^{3} L_{i} w_{i}
$$

$$
\begin{gathered}
\mathrm{g}\left(w_{1}, w_{2}, w_{3}\right)=\frac{T_{t}}{T_{1}}-0.75 \leq 0 \\
0.4 m \leq w_{1}, w_{2}, w_{3} \leq 0.8 m
\end{gathered}
$$

where $L_{i}$ is the length of the $i$ th shear wall. Equation (8) specifies that the ratio of the first torsional and translational periods to be less than or equal to 0.75 .

To create an RBF metamodel of Eq. (5) or (8), thirty-one sample points were generated using Latin hypercube sampling [11]. The structure was modeled using FE software package SAP2000 [12] at these sample points and modal analyses were conducted to obtain the modal information. The optimization results of the two formulations are shown in Table 1. It is found that the optimal shear wall thickness $w_{2}$ and $w_{3}$ are $0.4 \mathrm{~m}$ for both formulations. The optimal thickness $w_{l}=0.8 \mathrm{~m}$ is listed for formulation 1 and $w_{l}=0.441 \mathrm{~m}$ for formulation 2, respectively. To verify the optimal designs of the two formulations, two additional FE analyses were performed with the optimal shear wall thicknesses. It can be seen that the relative errors are $3.3 \%$ and $0.7 \%$ for the two formulations, which represent reasonable accuracy for this problem. Therefore, no additional sample points were created or used in the design optimization of this example.

Table 1: Optimal design results.

\begin{tabular}{|l|c|c|c|c|}
\hline & $\begin{array}{c}\text { 1st optimization } \\
\text { formulation }\end{array}$ & $\begin{array}{c}\text { 2nd optimization } \\
\text { formulation }\end{array}$ & $\begin{array}{c}\text { Lower } \\
\text { bound }\end{array}$ & $\begin{array}{c}\text { Upper } \\
\text { bound }\end{array}$ \\
\hline$w_{1}(\mathrm{~m})$ & 0.800 & 0.441 & 0.400 & 0.800 \\
\hline$w_{2}(\mathrm{~m})$ & 0.400 & 0.400 & 0.400 & 0.800 \\
\cline { 1 - 3 }$w_{3}(\mathrm{~m})$ & 0.400 & 0.400 & 0.400 & 0.800 \\
\cline { 1 - 3 }$T_{t} / T_{1}(\mathrm{RBF})$ & 0.647 & 0.750 & \multicolumn{2}{|}{} \\
\cline { 1 - 3 }$T_{t} / T_{1}(\mathrm{FE})$ & 0.669 & 0.745 & & \\
\cline { 1 - 3 }$T_{t} / T_{1}(\%$ error $)$ & $3.3 \%$ & $0.7 \%$ & & \\
\cline { 1 - 3 }
\end{tabular}

\section{Concluding Remarks}

A structural optimization approach was developed based on augmented RBF metamodels and a gradient-based optimization algorithm. The method was applied to the design optimization of an RC building to reduce torsional responses. FE analyses were performed to obtain the building modal information. A metamodeling method was applied to approximate the structural responses, and used in design optimization. The method worked well for the example problem and it provides an effective approach for design optimization of complex structures when expensive FE analyses and detailed user-interface programing are required for a nested application of gradient-based optimization. This method shall be further studied and applied to other complex structural engineering problems.

\section{References}

[1] U. Kirsch and G. I. N. Rozvany, "Alternative formulations of structural optimization,” Struct. Multidisc. Optim., vol. 7, pp. 32-41, 1994.

[2] J. S. Arora and Q. Wang, "Review of formulations for structural and mechanical system optimization," Struct. Multidisc. Optim., vol. 30, no. 4, pp. 251-272, 2005. 
[3] C.-M. Chan and Q. Wang, "Nonlinear stiffness design optimization of tall reinforced concrete buildings under service loads," J. Struct. Eng., ASCE, vol. 132, no. 6, pp. 978-990, 2006.

[4] X.-K. Zou and C.-M. Chan, "Optimal seismic performance-based design of reinforced concrete buildings using nonlinear pushover analysis," Engineering Structures, vol. 27, pp. 1289-1302, 2005.

[5] B. D. Youn and K. K. Choi, "A new response surface methodology for reliability-based design optimization," Computers \& Structures, vol. 82, pp. 241-256, 2004.

[6] J. Bi, H. Fang, Q. Wang and X. Ren, "Modeling and optimization of foam-filled thin-walled columns for crashworthiness designs," Finite Elements in Analysis and Design, vol. 46, no. 9, pp. 698-709, 2010.

[7] R. Jin, W. Chen and T. W. Simpson, "Comparative studies of metamodeling techniques under multiple modeling criteria," Struct. Multidisc. Optim., vol. 23, pp. 1-13, 2001.

[8] H. Fang, M. Rais-Rohani, Z. Liu and M. F. Horstemeyer, "A comparative study of metamodeling methods for multiobjective crashworthiness optimization," Computers \& Structures, vol. 83, no. 25-26, pp. 2121-2136, 2005.

[9] H. Fang and Q. Wang, "On the effectiveness of assessing model accuracy at design points for radial basis functions," Communications in Numerical Methods in Engineering, vol. 24, no. 3, pp. 219-235, 2008.

[10] Microsoft. Microsoft Excel 2013. Redmond, WA: Microsoft Corporation, 2013.

[11] D. C. Montgomery, Design and Analysis of Experiments. New York: John Wiley \& Sons, Inc., 2001.

[12] Computer and Structures, Inc. (CSI). SAP2000 Software, V15. Berkeley, California: Computer and Structures, Inc., 2011. 